\title{
Computer-Aided Detection of Lung Nodules in Chest X-Rays using Deep Convolutional Neural Networks
}

\author{
(D) Murat UÇAR ${ }^{1}$, (DEmine UÇAR ${ }^{2}$ \\ ${ }^{1}$ Department of Management Information Systems, İskenderun Technical University, Central Campus, \\ İskenderun, 31200, Hatay, Turkey, murat.ucar@ iste.edu.tr \\ ${ }^{2}$ Corresponding Author; Department of Management Information Systems, İskenderun Technical University, \\ Central Campus, İskenderun, 31200, Hatay, Turkey, emine.ucar@ iste.edu.tr, +90 5053963393
}

Received: 11/03/2019; Revision; 13/03/2019 Accepted; 20/03/2019 Published online; 25/04/2019

\begin{abstract}
Chest X-Rays are most accessible medical imaging technique for diagnosing abnormalities in the heart and lung area. Automatically detecting these abnormalities with high accuracy could greatly enhance real world diagnosis processes. In this study we aim to improve the accuracy of convolutional deep learning by using Laplacian of Gaussian filtering. In this study, we have used the publicly available Japanese Society of Radiological Technology dataset including 247 radiograms. For improving the performance of convolutional neural networks we used LoG filter and also we used an advanced version of AlexNet and GoogleNet to compare our results. The results indicated that, convolutional neural network with Laplacian of Gaussian filter model produced the best results with $82.43 \%$ accuracy. Convolutional neural network with Laplacian of Gaussian filter model is followed by convolutional neural network with an accuracy of $72.97 \%$, followed by GoogleNet model with an accuracy of $68.92 \%$. Out of the four model types utilized, the AlexNet model produced the lowest accuracy with a value of $64.86 \%$. The results obtained here demonstrate that the pre-processing technique like Laplacian of Gaussian filter can improve the accuracy.
\end{abstract}

Keywords: biomedical image processing, lung nodules detection, deep convolutional neural network

\section{Akciğer Nodüllerinin Göğüs Röntgenlerinden Derin Evrişimsel Sinir Ağları Kullanılarak Bilgisayar Destekli Tespiti}

\section{$\ddot{\mathbf{O} z}$}

Gögüs röntgenleri, kalp ve akciğerlerdeki anormallikleri teşhis etmek için en kolay erişilebilir tıbbi görüntüleme tekniğidir. Bu anormallikleri otomatik olarak yüksek hassasiyetle tespit etmek gerçek hayattaki teşhis süreçlerini büyük ölçüde artırmaktadır. Bu çalışmada, Gauss Laplace filtresini (LoG) kullanarak evrişimsel derin öğrenmenin doğruluk değerini arttırmayı amaçladık. Çalışmada, kamuya açık bir şekilde sunulan Japon Radyoloji Teknolojileri Derneğine ait 247 göğüs röntgeni görüntüsü kullanılmıştır. Evrişimsel sinir ağlarının performansını arttırmak için LoG filtresini ve daha sonra sonuçlarımızı karşılaşıtırmak için AlexNet ve GoogleNet modellerinin gelişmiş bir versiyonunu kullandık. Sonuçlar Gauss Laplace filtre modeli kullanılmış evrişimsel sinir ağının \% 82.43 doğrulukla en iyi sonuçları verdiğini göstermiştir. Bu modeli, \% 72.97 doğrulukla evrişimsel sinir ağı, \% 68.92 doğrulukla GoogleNet modeli izlemektedir. Kullanılan dört model türünden AlexNet modeli, \% 64.86 değeri ile en düşük doğruluğu üretmiş̧tir. Burada elde edilen sonuçlar, görüntü ön işleme tekniklerinden Gauss Laplace filtresinin doğruluğu artırabileceğini göstermektedir.

Anahtar Kelimeler: Biyomedikal görüntü işleme, akciğer nodülü tespiti, derin evrişimsel sinir ağı

\section{Introduction}

The chest $\mathrm{x}$-ray is the most commonly performed diagnostic $\mathrm{x}$-ray examination. A chest $\mathrm{x}$-ray produces images of the heart, lungs, blood vessels and the bones of the spinal cord and chest. An x-ray is a noninvasive medical test that helps doctors diagnose and treat medical conditions. X-rays are the oldest 
and most commonly used form of medical imaging modality. Computer Aided Diagnosis (CAD) is system that assists doctors in the interpretation of medical images. Development of a CAD system for the evaluation of medical images would increase the productivity of physicians and accessibility of better healthcare services in remote areas.

In recent time, deep convolutional neural network (DCNN) has gained popularity given its excellent performance in different image recognition challenges, such as image classification [1-4] and semantic segmentation [5-7]. DCNN is also applied in many medical image processing tasks [8-12] recently.

Lung cancer is the primary cause of tumor deaths in most countries and early diagnosis has an important value. In this work, we report DCNN based detection of lung nodules in chest X-Rays on the publicly available JSRT dataset. The paper is organized as follows. In section 2 we overview of the related work. In section 3, we describe the dataset and analysis method. Then in section 4, we present our results and compare the result obtained by this network with some other methodologies. Finally we discuss about the lacking and future possibilities of the presented network.

\section{Related Work}

Over the past decades, the volume of clinical data in machine-readable form has increased, especially in medical imaging. While previous generations of algorithms have sought to use of this highdimensional data effectively, modern neural networks have been successful at such tasks.

Convolutional Neural Networks (CNNs) have been recently employed to solve problems from medical image analysis fields. CNNs were first proposed by LeCun et al. in 1998 [13]. Ronneberger et al. built a more elegant architecture which is called UNets and developed an architecture based on data augmentation for using the available annotated samples more efficiently. The UNet architecture achieved very good performance on different biomedical segmentation applications [14]. Milletari et al. proposed a fully convolutional neural network to 3D image segmentation. They trained CNN end-toend on MRI volumes depicting prostate, and their network has learned to predict segmentation for the whole volume at once. According to the experimental evaluation that their approach has achieved good performances on challenging test data [15]. Long et al. showed that convolutional networks by themselves, trained end-to-end, pixels to pixels, exceed the state-of-the-art in semantic segmentation [16]. Esteva et al. trained a single CNN for skin cancer classification and the CNN demonstrated a good performance as dermatologists' opinions. [17]. Gulshan et al. proposed an algorithm based on deep learning for automated detection of diabetic retinopathy and diabetic macular edema in retinal fundus photographs. Their research had high sensitivity and specificity for detecting referable diabetic retinopathy [18]. Lakhani \& Sundaram used deep convolutional neural networks for pulmonary tuberculosis detection in chest radiographs and they classified tuberculosis at chest X-rays with an AUC of 0.99 [19]. Huang et al. (2017b) used convolutional neural networks for lung cancer diagnosis with chest CTs [20]. Rajpurkar et al. used CheXNet model to detect automatically many lung diseases [21]. Gordienko et al. demonstrated efficiency of dimensionality reduction performed by lung segmentation, bone shadow exclusion, and t-distributed stochastic neighbor embedding (t-SNE) techniques for analysis of 2D CXR of lung cancer patients [22].

\section{Materials and Methods}

Chest X-Rays were used in the proposed diagnosis system. Preprocessing stage of the developed system consists of Laplacian of Gaussian filtering process. We preferred the Laplacian of Gaussian filter for improving the performance of convolutional deep learning model and also we used an advanced version of AlexNet and GoogleNet to compare our results. Within the scope of our study, the steps and methods used in processing the Chest X-Ray images and detection of lung nodules are shown schematically in Figure 1. 


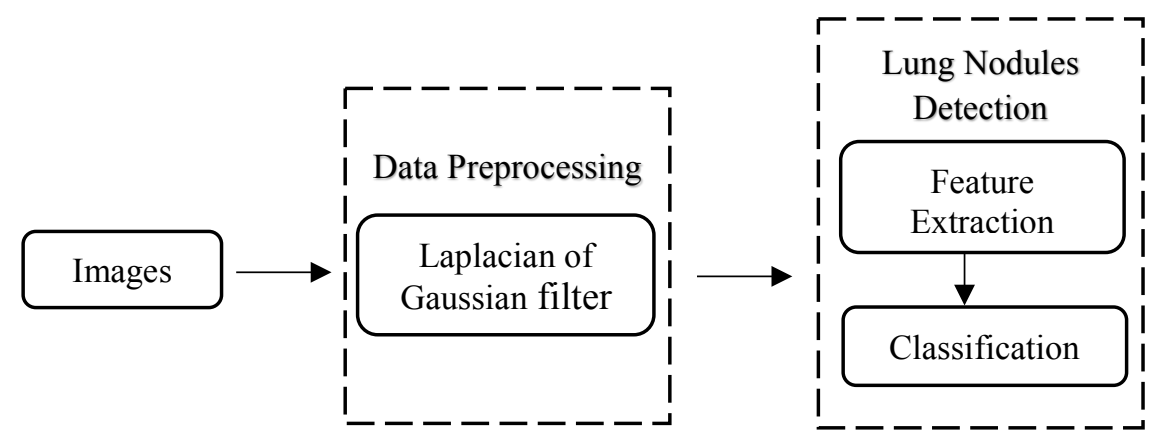

Figure 1 The Steps and Methods used in Study

\subsection{Dataset}

In this study we used the public Standard Digital Image Database, collected by the Japanese Society of Radiological Technology (JSRT). The JSRT database was formed to provide a public set of annotated images which would be available for research purposes, training and education. As fully described in [23], the JSRT database is composed of $24714 \times 14$ inch postero-anterior chest radiographs, each one being digitized into a $2048 \times 2048 \times 12$ bit matrix; 154 images contain a single 1 ung nodule, and the remaining 93 are negative for nodules. The nodule size ranged from 5 to $40 \mathrm{~mm}$ with an average size of $24.6 \mathrm{~mm}$. The patient age ranged from 21 to 79 with an average of 59.68. There were 81 female patients and 83 patients with malignant nodules.

\subsection{Laplacian of Gaussian Filter}

Laplacian of Gaussian as can be formulated as the following equation is a filter that can be used to find rapid change in pixels values in image data. The Gaussian in LoG can smooth the image and reduce the impact of noise on it. It can also offset the influence of increasing noise caused by the second derivative of the Laplacian. The $\sigma$ can actually control the amount of filtering [24]:

$$
\nabla^{2} G(x, y)=\frac{1}{2 \pi \sigma^{4}}\left(2-\left(\frac{x^{2}+y^{2}}{\sigma^{2}}\right)\right) e^{-\left(x^{2}+y^{2}\right) / 2 \sigma^{2}}
$$

Laplacian of Gaussian can be used as appropriate features of images by finding the significiant variation in the pixels instead of using all the pixel values that can contain meaningless data [25]. Figure 2 shows the example of the result of applying the LoG filter to our database.

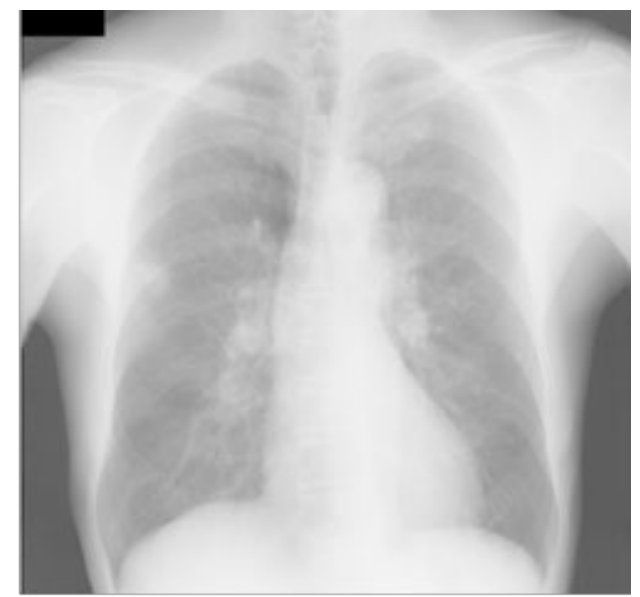

a)

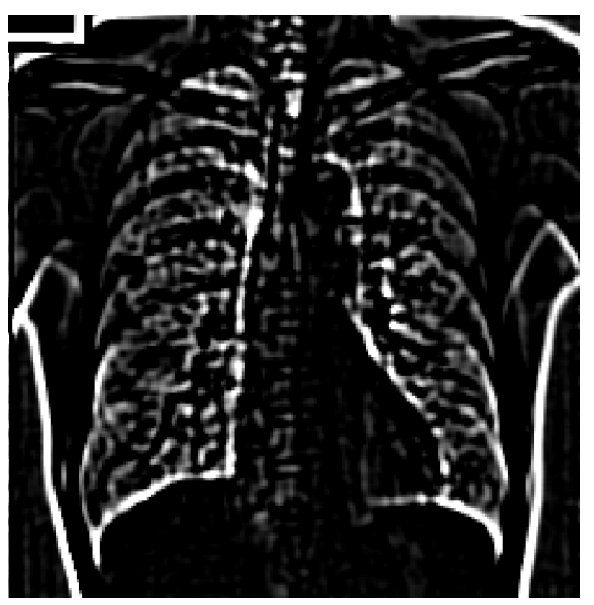

b)

Figure 2 Example of the Result of Applying the LoG filter to Our Database a) Original Image b) Image with LoG Filter. 


\subsection{Convolutional Neural Networks}

CNNs are feed-forward ANN inspired by biological processes and designed to recognize patterns directly from pixel images (or other signals), by incorporating both feature extraction and classification. A typical CNN involves four types of layers: convolutional, activation, pooling and fully-connected (or dense) layers [26]. In our study we used 7 layers for deep concolutional neural network model. These are input layer, convolution $2 \mathrm{~d}$ layer, relu layer, max pooling $2 \mathrm{~d}$ layer, full connected layer, softmax layer and classification layer. In the convolution layer, a $5 \times 5$ filter was applied to the input image and the output was normalized by the ReLU activation function. After the ReLU layer, it was performed in 2 squares for the stride operation in the MaxPooling layer. The initial learning rate is 0.0001 as a starting point. $70 \%$ of the data set was used for training and the remaining $30 \%$ was used for testing. Our proposed DCNN model is shown in Figure 3.

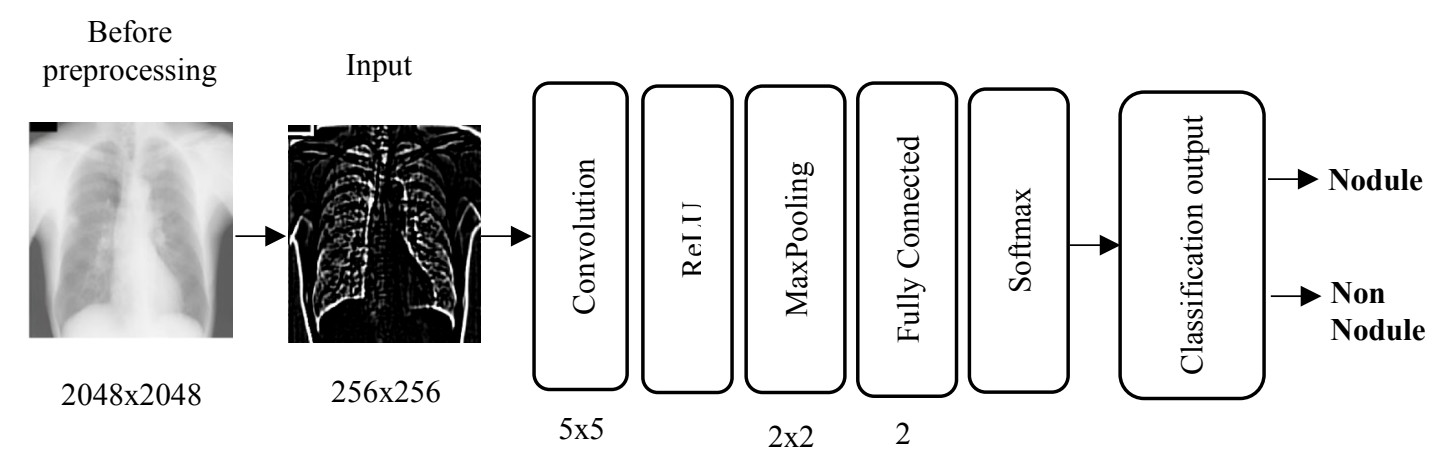

Figure 3 DCNN Architecture of Proposed Method

\subsection{CNN Architectures}

AlexNet made CNNs popular in Computer Vision. It is composed of 5 convolutional layers followed by 3 fully connected layers [27]. It was developed by Alex Krizhevsky et al. and won ImageNet ILSVRC challenge in 2012 [1]. During this competition it produced the best results, top-1 and top-5 error rates of $37.5 \%$ and $17.0 \%$.

GoogLeNet was invented by Szegedy et al. from Google that was the winner of ILSVRC 2014 [3]. Its main contribution was the development of an inception module, which concatenates filters of different sizes and dimensions into a single new filter. Overall, GoogLeNet has two convolution layers, two pooling layers, and nine "Inception" layers. Each "Inception" layer consists of six convolution layers and one pooling layer. GoogLeNet is the current state-of-the-art CNN architecture for the ILSVRC challenge, where it achieved 5.5\% top-5 classification error on the ImageNet challenge, compared to AlexNet's $15.3 \%$ top-5 classification error [28].

\section{Results}

In this section, the performance of the proposed method is evaluated via its effect on the nodule detection in chest radiographs. A compare with other existing methods are also provided. The DCNN was trained in MATLAB machine learning framework on the dataset to predict presence (154 images) or absence (93 images) of nodule. Several training and validation runs for the DCNN on CXR images from JSRT database were performed. The results of our runs are shown in Figure 4.

The evident over-training can be observed after comparison of training and validation results, where the averaged and smoothed value of training accuracy is going with epochs to the theoretical maximum of 1 and training loss is going to 0 . As the results indicate, DCNN with LoG filter model produced the best results with $82.43 \%$ accuracy. DCNN with LoG filter model is followed by DCNN with an accuracy of $72.97 \%$, followed by GoogleNet model with an accuracy of $68.92 \%$. Out of the four model types 
utilized, the AlexNet model produced the lowest accuracy with a value of $64.86 \%$ (Table 1). The results obtained here demonstrate that the pre-processing technique LoG filter can improve the accuracy.

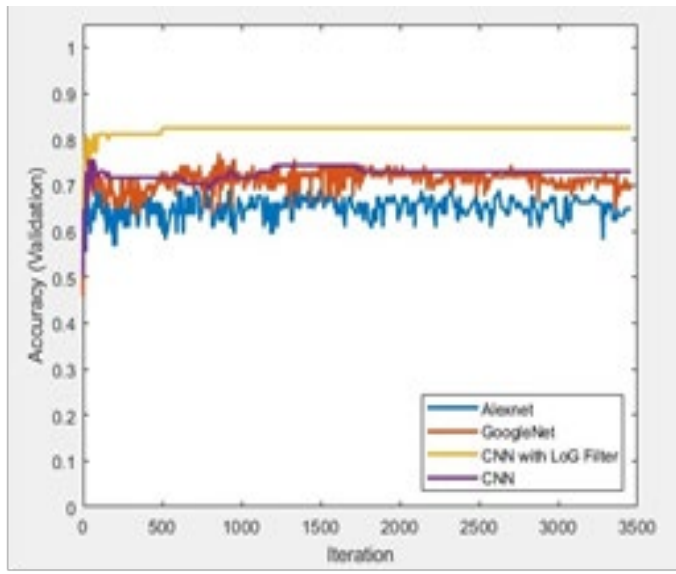

a)

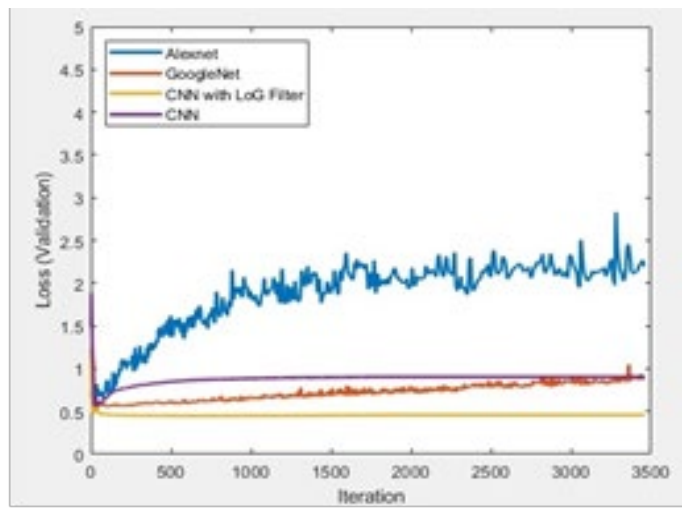

c)

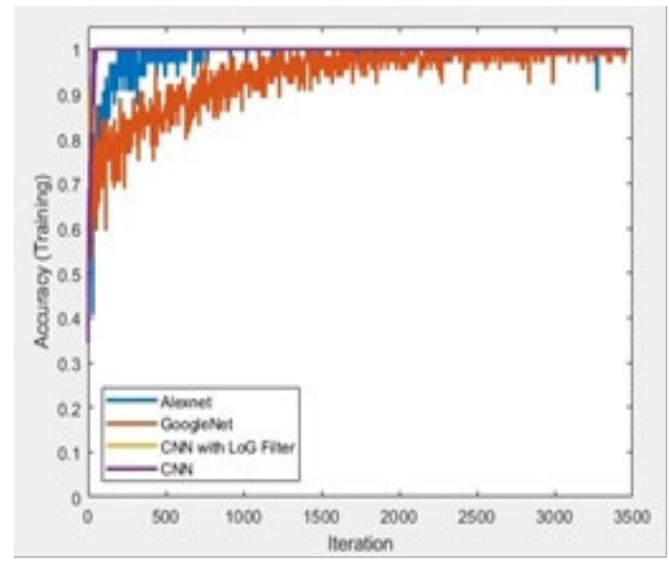

b)

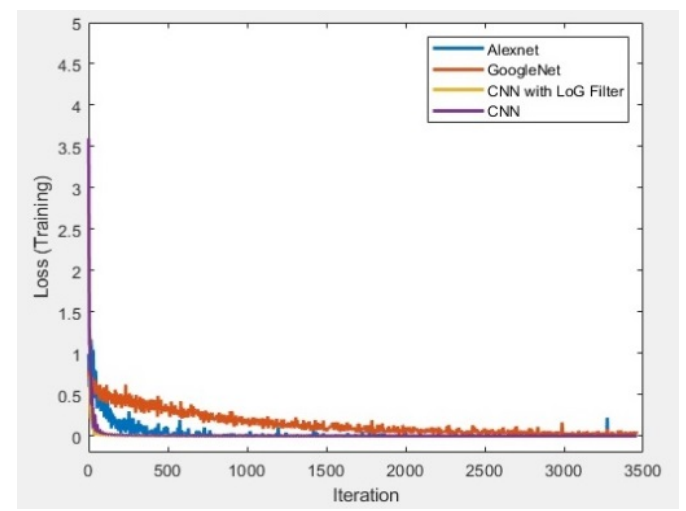

d)

Figure 4 A Comparison of Nodule Detection Performance with Different Models a) Validation Accuracy b) Training Accuracy c) Validation Loss d)Training Loss.

Table 1 Comparison of Used Models on Diagnosis of the Lung Nodules

\begin{tabular}{|l|r|r|}
\hline Classifier & Accuracy & Epoch \\
\hline Deep Convolutional Neural Network & $72.97 \%$ & 720 \\
\hline $\begin{array}{l}\text { Deep Convolutional Neural Network } \\
\text { with LoG filter }\end{array}$ & $\mathbf{8 2 . 4 3 \%}$ & 770 \\
\hline AlexNet & $64.86 \%$ & 731 \\
\hline GoogleNet & $68.92 \%$ & 756 \\
\hline
\end{tabular}

\section{Conclusion}

In this paper, we proposed an image processing method based on the Laplacian of a Gaussian filter to detect lung nodules in CXRs. The proposed method can effectively improve image contrast to help doctors detect lung cancer. And also experimental results demonstrate that the proposed method is efficient and effective for the CNNs. 
As future work, we will use a larger real dataset for training and especially we will try other preprocessing techniques on validation.

\section{References}

[1] A. Krizhevsky, I. Suskever and G.E. Hinton, "ImageNet classification with deep convolutional neural networks, " in International Conference on Neural Information Processing Systems, 2012, pp. 1106-1114.

[2] K. Simonyan and A. Zisserman, "Very deep convolutional networks for large-scale image recognition, " In ICLR, 2015.

[3] C. Szegedy, W. Liu, Y. Jia, P. Sermanet, S. Reed, D. Anguelov, D. Erhan, V. Vanhoucke and A. Rabinovich, "Going deeper with convolutions, " in IEEE Conference on Computer Vision and Pattern Recognition, 2015, pp. 1-9.

[4] K. He, X. Zhang, S. Ren and J. Sun, "Deep residual learning for image recognition, " in IEEE Conference on Computer Vision and Pattern Recognition, 2016, pp. 770-778.

[5] M. Mostajabi, P. Yadollahpour and G. Shakhnarovich, "Feedforward semantic segmentation with zoom-out features, " in IEEE Conference on Computer Vision and Pattern Recognition, 2015, pp. 33763385 .

[6] H. Noh, S. Hong and B. Han, "Learning deconvolution network for semantic segmentation, " in Proceedings of the 2015 IEEE International Conference on Computer Vision, 2015, pp. 1520-1528.

[7] L.C. Chen, G. Papandreou, I. Kokkinos, K. Murphy and A. L. Yuille, "Semantic image segmentation with deep convolutional nets and fully connected CRFs, " Computer Science, vol. 4, pp. 357-361, 2016.

[8] B.V. Ginneken, A. A. A. Setio, C. Jacobs and F. Ciompi, "Off-the-shelf convolutional neural network features for pulmonary nodule detection in computed tomography scans, in IEEE International Symposium on Biomedical Imaging, 2015, pp. 286-289.

[9] L. Rongjian, Z. Wenlu, S. Heung-Il, W. Li, L. Jiang, S. Dinggang and J. Shuiwang, "Deep learning based imaging data completion for improved brain disease diagnosis, " in International Conference on Medical Image Computing and Computer-Assisted Intervention, 2014, pp. 305-312.

[10] H. R. Roth, L. Lu, J. Liu, J. Yao, A. Seff, K. Cherry, L. Kim and R.M. Summers, "Improving computer-aided detection using convolutional neural networks and random view aggregation, " IEEE Transactions on Medical Imaging, vol. 35, no. 5, pp. 1170-1181, 2016.

[11] Y. Bar, I. Diamant, L. Wolf, S. Lieberman, E. Konen and H.Greenspan, "Chest pathology detection using deep learning with non-medical training, " in IEEE International Symposium on Biomedical Imaging, 2015, pp. 294-297.

[12] H. C. Shin, K. Roberts, L. Lu, D. Demnerfushman, J. Yao and R. M. Summers, "Learning to read chest X-rays: Recurrent neural cascade model for automated image annotation, " in IEEE Conference on Computer Vision and Pattern Recognition, 2016, pp. 2497-2506. 
[13] Y. LeCun, L. Bottou, Y. Bengio and P. Haffner, "Gradient-based learning applied to document recognition, " Proceedings of the IEEE, vol. 86, no. 11, pp. 2278-2324, 1998.

[14] O. Ronneberger, P. Fischer and T. Brox, U-net: "Convolutional networks for biomedical image segmentation, " in International Conference on Medical Image Computing and Computer-Assisted Intervention, 2015, pp. 234-241.

[15] F. Milletari, N. Navab and S. A. Ahmadi, "V-net: Fully convolutional neural networks for volumetric medical image segmentation, " in 2016 Fourth International Conference on 3D Vision, 2016, pp. 565-571.

[16] J. Long, E. Shelhamer and T. Darrell, "Fully convolutional networks for semantic segmentation, " in IEEE Conference on Computer Vision and Pattern Recognition, 2015, pp. 3431-3440.

[17] A. Esteva, B. Kuprel, R.A. Novoa, J. Ko, S. M. Swetter, H.M. Blau and S. Thrun, "Dermatologistlevel classification of skin cancer with deep neural networks, " Nature, vol. 542, no. 7639, pp. 115-118, 2017.

[18] V. Gulshan, L. Peng, M. Coram, M.C. Stumpe, D.Wu, A. Narayanaswamy, S. Venugopalan, K.Widner, T. Madams, J Cuadros, et al., "Development and validation of a deep learning algorithm for detection of diabetic retinopathy in retinal fundus photographs, "Jama, vol. 316, no.22, pp. 2402-2410, 2016.

[19] P. Lakhani and S. Baskaran, "Deep learning at chest radiography: Automated classification of pulmonary tuberculosis by using convolutional neural networks, " Radiology, vol. 284, no.2, pp. 574$582,2017$.

[20] P. Huang, S. Park, R. Yan, J. Lee, L.C. Chu, C.T. Lin, A. Hussien, J. Rathmell, B. Thomas, C.Chen, et al., "Added value of computer-aided CT image features for early lung cancer diagnosis with small pulmonary nodules: A matched case-control study, " Radiology, vol. 286, no.2, pp. 286-295, 2017.

[21] P. Rajpurkar, J. Irvin, K. Zhu, B. Yang, H. Mehta, T. Duan, D. Ding, A. Bagul, C. Langlotz, K.Shpanskaya, M. P. Lungren and Y. Ng. Andrew, "CheXNet: Radiologist-Level Pneumonia Detection on Chest X-Rays with Deep Learning, " in IEEE Conference on Computer Vision and Pattern Recognition, 2017.

[22] Y. Gordienko, Y. Kochura, O. Alienin, O. Rokovyi, S. Stirenko, P. Gang, J. Hui and W. Zeng, "Dimensionality Reduction in Deep Learning for Chest X-Ray Analysis of Lung Cancer," in International Conference on Advanced Computational Intelligence, 2018.

[23] J. Shiraishi, S. Katsuragawa, J. Ikezoe, T. Matsumoto, T. Kobayashi, K. Komatsu, M. Matsui, H. Fujita, Y. Kodera and K. Doi, "Development of a digital image database for chest radiographs with and without a lung nodule, " AJR Am J Roentgenol, vol.174, no.1, pp. 71-74, 2000.

[24] G. E. Sotak, Jr. and K. L. Boyer, "The Laplacian-of-Gaussian kernel: a formal analysis and design procedure for fast, accurate convolution and full-frame output, " Comput.Vis.Gr. Image Process, vol. 48, no. 2, pp. 147-189, 1989. 
[25] A. Huertas and G. Medioni, "Detection of intensity changes with subpixel accuracy using Laplacian-Gaussian masks, " IEEE Trans. Pattern Anal. Mach. Intell, vol. 8, no.5, pp. 651-664, 1986.

[26] M. Anthimopoulos, S. Christodoulidis, L. Ebner, A. Christe, and S. Mougiakakou, "Lung pattern classification for interstitial lung diseases using a deep convolutional neural network, " IEEE Trans. Med. Imaging, vol. 35, no.5, pp. 1207-1216, 2016.

[27] M. Çoşkun, Ö. Yıldırım, A. Uçar, and Y. Demir, "An overview of popular deep learning methods," European Journal of Technique, vol. 7, no. 2, pp. 165-176, 2017.

[28] H.C. Shin, H.R Roth, M. Gao, et al., "Deep convolutional neural networks for computer-aided detection: CNN architectures, dataset characteristics and transfer learning," IEEE Trans. Med. Imaging, vol. 35, no. 5, pp. 1285-1298, 2016. 